\title{
Oscillation criteria for neutral second-order half-linear differential equations with applications to Euler type equations
}

\section{Simona Fišnarová and Robert Mař́k ${ }^{*}$}

${ }^{\text {*Correspondence: }}$

marik@mendelu.cz

Department of Mathematics,

Mendel University in Brno,

Zemědělská 1, Brno, 613 00, Czech

Republic

\begin{abstract}
We study the second-order neutral delay half-linear differential equation $\left[r(t) \Phi\left(z^{\prime}(t)\right)\right]^{\prime}+q(t) \Phi(x(\sigma(t)))=0$, where $\Phi(t)=|t|^{\alpha-1} t, \alpha \geq 1$ and $z(t)=x(t)+p(t) x(\tau(t))$. We use the method of Riccati type substitution and derive oscillation criteria for this equation. By an example of the neutral Euler type equation we show that the obtained results are sharp and improve the results of previous authors. Among others, we improve the results of Sun et al. (Abstr. Appl. Anal. 2012:819342, 2012) and discuss also the case when $\sigma \circ \tau \neq \tau \circ \sigma$.
\end{abstract}

MSC: Primary 34K11; secondary 34K40

Keywords: half-linear differential equation; oscillation criteria; Riccati technique; delay equation; neutral equation; Euler type equation

\section{Introduction}

In the paper we study the equation

$$
\left[r(t) \Phi\left(z^{\prime}(t)\right)\right]^{\prime}+q(t) \Phi(x(\sigma(t)))=0, \quad z(t)=x(t)+p(t) x(\tau(t))
$$

where $\Phi(t)=|t|^{\alpha-1} t$ is the power type nonlinearity, $\alpha \geq 1$, which ensures that the function $\Phi(\cdot)$ is a convex function on $(0, \infty)$. The coefficients $r$ and $p$ are subject of usual conditions $r \in C^{1}\left(\left[t_{0}, \infty\right), \mathbb{R}^{+}\right), p \in C^{1}\left(\left[t_{0}, \infty\right), \mathbb{R}_{0}^{+}\right)$and the coefficient $q$ is positive $q \in C\left(\left[t_{0}, \infty\right), \mathbb{R}^{+}\right)$. Further we suppose that the deviating arguments are unbounded and sufficiently smooth functions: $\tau \in C^{2}\left(\left[t_{0}, \infty\right), \mathbb{R}\right), \tau^{\prime}(t)>0, \lim _{t \rightarrow \infty} \tau(t)=\infty, \sigma \in C^{1}\left(\left[t_{0}, \infty\right), \mathbb{R}\right), \sigma^{\prime}(t)>0$, $\lim _{t \rightarrow \infty} \sigma(t)=\infty$ and the deviating arguments from the differential term and potential satisfy either $\sigma(\tau(t))=\tau(\sigma(t))$ or $\sigma(\tau(t)) \geq \tau(\sigma(t))$ (in the latter case we use stronger condition on the coefficient $p(t)$ and the conclusion is weaker than in the commutative case).

By the solution of (1) we understand any differentiable function $x(t)$ which does not identically equal zero eventually, such that $r(t) \Phi\left(z^{\prime}(t)\right)$ is differentiable and (1) holds for large $t$.

The solution of (1) is said to be oscillatory if it has infinitely many zeros tending to infinity. Equation (1) is said to be oscillatory if all its solutions are oscillatory. In the opposite case, i.e., if there exists an eventually positive solution of (1), (1) is said to be nonoscillatory.

The neutral equations naturally arise in the mathematical models where the rate of the growth depends not only on the current state and the state in the past, but also on the

@2014 Fišnarová and Mařík; licensee Springer. This is an Open Access article distributed under the terms of the Creative Commons Attribution License (http://creativecommons.org/licenses/by/2.0), which permits unrestricted use, distribution, and reproduction in any medium, provided the original work is properly cited. 
rate of change in the past. The paper [1] suggests to imagine a child, which begins to grow more rapidly at the age of about 12 years, growing more and more rapidly until a certain height is approached, at which time there is a rapid slowing of the growth, stopping at the adult height dictated by genes. This process can easily be modeled by neutral equation. Similarly, the paper [2] suggests one to use a logistic neutral differential equation to model a population of Daphnia magna. If in the system modeled by first-order neutral differential equation the mature individuals produce some toxin which inhibits the rate the growth and if the production of this toxin is constant per capita and unit time, then the rate of the growth is inhibited by the term like $\int^{t} x(\sigma(s)) \mathrm{d} s$ and we naturally obtain the second-order neutral differential equation.

This research is motivated by the paper [3], where the main results of [3] are illustrated by an example of the equation

$$
\left[\Phi\left(\left(x(t)+p(t) x\left(\lambda_{1} t\right)\right)^{\prime}\right)\right]^{\prime}+\frac{\beta}{t^{\alpha+1}} \Phi\left(x\left(\lambda_{2} t\right)\right)=0
$$

with $0 \leq p(t) \leq p_{0}<\infty, \alpha \geq 1, \beta>0, \lambda_{1} \in(0,1)$.

If $0<\lambda_{2} \leq \lambda_{1}$, then (2) is proved to be oscillatory if (see [3, Example 5.2])

$$
\beta>\frac{2^{\alpha-1} \alpha^{\alpha+1}}{(\alpha+1)^{\alpha+1} \lambda_{2}^{\alpha}}\left(1+\frac{p_{0}^{\alpha}}{\lambda_{1}}\right)
$$

If $\lambda_{2} \in\left[\lambda_{1}, \infty\right)$, then (2) is proved to be oscillatory if (see [3, Example 5.4])

$$
\beta>\frac{2^{\alpha-1} \alpha^{\alpha+1}}{(\alpha+1)^{\alpha+1} \lambda_{1}^{\alpha}}\left(1+\frac{p_{0}^{\alpha}}{\lambda_{1}}\right)
$$

Note that if we put $\lambda_{1}=\lambda_{2}=1$ and $p_{0}=0$, both oscillation constants (3) and (4) are worse than the constant

$$
\beta_{E}:=\frac{\alpha^{\alpha+1}}{(\alpha+1)^{\alpha+1}}
$$

which is well known to be an optimal oscillation constant for the Euler type equation

$$
\left[\Phi\left(x^{\prime}(t)\right)\right]^{\prime}+\frac{\beta}{t^{\alpha+1}} \Phi(x(t))=0
$$

see [4, Chapter 1.4.2]. The aim of this paper is to develop sharper results than those of [3]. The main idea is to use the classical approach based on Riccati type inequality. Due to the neutral nature of (1) we have to work with (1) and with the same equation shifted from $t$ to $\tau(t)$. In contrast to the results of some other authors $[3,5,6]$, we do not simply sum up the arising Riccati equations, but we develop an advanced technique based on suitable linear combination and careful comparison of $q(t)$ and $q(\tau(t))$. We show that an application of this technique allows to remove the above mentioned disadvantages of the paper [3] and allows to derive sharper results comparing the results published in the literature. Finally, we also discuss the case when the usual assumption $\sigma \circ \tau=\tau \circ \sigma$ is broken. This step opens applications to the neutral delay differential equations with proportional delay $\sigma$ and constant delay $\tau$. 
The paper is organized as follows. In the following section we formulate inequalities which are used to prove the main results. Section 3 contains main results of the paper and examples which prove that we provide sharp oscillation constant for Euler type differential equation. These criteria are expressed in terms of positive mutually conjugate numbers $l$ and $l^{*}$, the multiplicative factor $\varphi$, and a function $Q(t)$.

\section{Preliminary results}

First we derive some technical lemmas - inequalities which are necessary to reduce second-order differential equation into a combination of two first-order Riccati type equations. Note the changes against [3] and other related papers.

(i) We use convex linear combination (6) instead of arithmetic mean in Lemma 2. To achieve this, we consider two positive mutually conjugate numbers $l$ and $l^{*}$, i.e. $l>1$ and $l^{*}=\frac{l}{l-1}$.

(ii) We relax the condition on the commutativity of the composition of $\sigma$ and $\tau$ if $x$ is an increasing function in Lemma 3.

(iii) We use new multiplicative factor $\varphi(t)$ in the definition of the function $Q(t)$ in Lemma 4. The factor $\varphi$ allows us to make terms involving $q(t)$ and $q(\tau(t))$ closer (or even equal, as in Examples 1 and 4 below) when looking for a smaller one.

As far as we know, these ideas have never been used in the context of Riccati technique even in the linear case $\alpha=1$ and in the section with main results we show that these points are crucial points of the paper which allow to derive sharper results than the results published in the literature.

Throughout the paper $A_{+}$denotes the positive part of $A$, i.e. $A_{+}=\max \{A, 0\}$.

Lemma 1 The following inequality holds for every $A$ and every $B \geq 0, \omega \geq 0$ :

$$
A \omega-B \omega^{\frac{\alpha+1}{\alpha}} \leq \frac{\alpha^{\alpha}}{(\alpha+1)^{\alpha+1}} A_{+}^{\alpha+1} \frac{1}{B^{\alpha}} .
$$

Proof The inequality is trivial if $A \leq 0$ and a special case of the Young inequality if $A>0$.

Lemma 2 The following inequality holds for $\alpha \geq 1$, positive mutually conjugate numbers $l, l^{*}$, and nonnegative $a$ and $b$ :

$$
\frac{1}{l} a^{\alpha}+\frac{1}{l^{*}} b^{\alpha} \geq\left(\frac{1}{l} a+\frac{1}{l^{*}} b\right)^{\alpha} .
$$

Proof The proof follows immediately from the convexity of $x^{\alpha}$.

Lemma 3 Suppose that either

$$
\sigma(\tau(t))=\tau(\sigma(t))
$$

or suppose that $x$ is an increasing function and

$$
\sigma(\tau(t)) \geq \tau(\sigma(t))
$$


The inequality

$$
l^{\alpha-1} x^{\alpha}(\sigma(t))+\left(l^{*}\right)^{\alpha-1} p^{\alpha}(\sigma(t)) x^{\alpha}(\sigma(\tau(t))) \geq z^{\alpha}(\sigma(t))
$$

holds for positive mutually conjugate numbers $l, l^{*}$, and every $t$ which satisfies $x(\sigma(t)) \geq 0$ and $x(\sigma(\tau(t))) \geq 0$.

Proof From the previous lemma using $a=l x(\sigma(t))$ and $b=l^{*} p(\sigma(t)) x(\sigma(\tau(t)))$ and also from the definition of $z(t)$ and the fact that $x(\sigma(\tau(t))) \geq x(\tau(\sigma(t)))$.

Lemma 4 Let $x$ be solution of (1). Suppose that either (7) holds or suppose that $x$ is an increasing function and (8) holds. The inequality

$$
l^{\alpha-1}\left[r(t) \Phi\left(z^{\prime}(t)\right)\right]^{\prime}+\left(l^{*}\right)^{\alpha-1} \varphi(t) \frac{p^{\alpha}(\sigma(t))}{\tau^{\prime}(t)}\left[r(\tau(t)) \Phi\left(z^{\prime}(\tau(t))\right)\right]^{\prime}+Q(t) z^{\alpha}(\sigma(t)) \leq 0,
$$

where

$$
Q(t)=\min \{q(t), \varphi(t) q(\tau(t))\}
$$

is valid for positive mutually conjugate numbers $l, l^{*}$, and a positive function $\varphi(t)$ whenever $x(\sigma(t))$ and $x(\sigma(\tau(t)))$ are nonnegative. Moreover, if there exist numbers $p_{0}$ and $\tau_{0}$ such that $p(t) \leq p_{0}<\infty, \tau^{\prime}(t) \geq \tau_{0}>0$, we have also

$$
l^{\alpha-1}\left[r(t) \Phi\left(z^{\prime}(t)\right)\right]^{\prime}+\left(l^{*}\right)^{\alpha-1} \varphi(t) \frac{p_{0}^{\alpha}}{\tau_{0}}\left[r(\tau(t)) \Phi\left(z^{\prime}(\tau(t))\right)\right]^{\prime}+Q(t) z^{\alpha}(\sigma(t)) \leq 0,
$$

whenever $x(\sigma(t))$ and $x(\sigma(\tau(t)))$ are nonnegative and $\left[r(\tau(t)) \Phi\left(z^{\prime}(\tau(t))\right)\right]^{\prime}$ is negative.

Proof To obtain the second term from the definition of $z$ we shift (1) from $t$ to $\tau(t)$ and multiply by $p^{\alpha}(\sigma(t))$. We get

$$
\frac{p^{\alpha}(\sigma(t))}{\tau^{\prime}(t)}\left[r(\tau(t)) \Phi\left(z^{\prime}(\tau(t))\right)\right]^{\prime}+q(\tau(t)) p^{\alpha}(\sigma(t)) x^{\alpha}(\sigma(\tau(t)))=0,
$$

where $z^{\prime}(\tau(t))=\left.\frac{\mathrm{d} z(s)}{\mathrm{d} s}\right|_{s=\tau(t)}$.

Now we multiply (1) by $l^{\alpha-1},(13)$ by $\varphi(t)\left(l^{*}\right)^{\alpha-1}$, and we add. We obtain

$$
\begin{aligned}
0 \geq & l^{\alpha-1}\left[r(t) \Phi\left(z^{\prime}(t)\right)\right]^{\prime}+\left(l^{*}\right)^{\alpha-1} \varphi(t) \frac{p^{\alpha}(\sigma(t))}{\tau^{\prime}(t)}\left[r(\tau(t)) \Phi\left(z^{\prime}(\tau(t))\right)\right]^{\prime} \\
& +\min \{q(t), \varphi(t) q(\tau(t))\}\left[l^{\alpha-1} x^{\alpha}(\sigma(t))+\left(l^{*}\right)^{\alpha-1} p^{\alpha}(\sigma(t)) x^{\alpha}(\sigma(\tau(t)))\right] .
\end{aligned}
$$

Now (10) follows from the definition of $Q$ and from (9). Inequality (12) follows from (10) and from the fact that $\left[r(\tau(t)) \Phi\left(z^{\prime}(\tau(t))\right)\right]^{\prime}$ is negative.

\section{Main results with applications to Euler type equation}

Now we are ready to prove the main results of the paper. The function $Q$ which appears in these criteria is a function defined by (11).

We will distinguish two cases: $\sigma(t) \leq \tau(t)$ and $\tau(t) \leq \sigma(t)$. Let us start with the first case. 
Theorem 1 Suppose that (7) and

$$
\int^{\infty} \frac{1}{r^{1 / \alpha}(t)} \mathrm{d} t=\infty
$$

hold. Further suppose that $\sigma(t) \leq t, \sigma(t) \leq \tau(t)$ and there exist positive mutually conjugate numbers $l, l^{*}$ and positive functions $\rho \in C^{1}\left(\left[t_{0}, \infty\right), \mathbb{R}^{+}\right), \varphi(t)$ such that

$$
\begin{aligned}
& \limsup _{t \rightarrow \infty} \int_{t_{0}}^{t} \rho(s) Q(s)-\frac{1}{(\alpha+1)^{\alpha+1}} \frac{\rho(s) r(\sigma(s))}{\left(\sigma^{\prime}(s)\right)^{\alpha}} \\
& \quad \times\left[l^{\alpha-1}\left(\frac{\rho_{+}^{\prime}(s)}{\rho(s)}\right)^{\alpha+1}+\left(l^{*}\right)^{\alpha-1} \frac{p^{\alpha}(\sigma(s)) \varphi(s)}{\tau^{\prime}(s)}\right. \\
& \left.\quad \times\left(\frac{\rho^{\prime}(s)}{\rho(s)}+\left(\frac{p^{\alpha}(\sigma(s)) \varphi(s)}{\tau^{\prime}(s)}\right)^{\prime} \frac{\tau^{\prime}(s)}{p^{\alpha}(\sigma(s)) \varphi(s)}\right)_{+}^{\alpha+1}\right] \mathrm{d} s=\infty .
\end{aligned}
$$

Then (1) is oscillatory.

Proof Suppose, by contradiction, that all of the assumptions of the theorem hold and there exists a solution $x(t)$ of $(1)$ and a number $t_{1}>t_{0}$ which satisfies

$$
\min \{x(t), x(\tau(t)), x(\sigma(t)), x(\tau(\sigma(t)))\}>0
$$

for every $t>t_{1}$.

Condition (14) ensures that the corresponding function $z$ is eventually increasing. In fact, from (1) we have

$$
\left(r(t) \Phi\left(z^{\prime}(t)\right)\right)^{\prime}=-q(t) \Phi(x(\sigma(t)))<0
$$

for $t \in\left(t_{1}, \infty\right)$. Hence $r(t) \Phi\left(z^{\prime}(t)\right)$ is decreasing and either

$$
\Phi\left(z^{\prime}(t)\right)>0 \quad \text { or } \quad \Phi\left(z^{\prime}(t)\right)<0
$$

for large $t$.

Suppose that there exists $T>t_{1}$ such that $\Phi\left(z^{\prime}(t)\right)<0$ for $t \geq T$. There exists a positive constant $M$ such that

$$
r(t) \Phi\left(z^{\prime}(t)\right)<-M<0
$$

and

$$
z^{\prime}(t)<-\Phi^{-1}(M) r^{-1 / \alpha}(t)
$$

for $t \geq T$. Integrating this inequality over the interval $(T, t)$ we get

$$
z(t) \leq z(T)-\Phi^{-1}(M) \int_{T}^{t} r^{-1 / \alpha}(s) \mathrm{d} s
$$


Letting $t \rightarrow \infty$ we have a negative upper bound for the function $z$ and large $t$. However, the positivity of both $x(t)$ and $x(\tau(t))$ implies positivity of $z$. This contradiction proves that $\Phi\left(z^{\prime}(t)\right)>0$ and $z^{\prime}(t)>0$ eventually.

Consequently, we will work on the interval $\left(t_{2}, \infty\right)$ where $t_{2}$ is such that

$$
\min \left\{x(t), x(\tau(t)), x(\sigma(t)), x(\sigma(\tau(t))), z^{\prime}(t), z^{\prime}(\tau(t)), z^{\prime}(\sigma(t)), z^{\prime}(\tau(\sigma(t)))\right\}>0
$$

for every $t>t_{2}$.

Define

$$
\omega(t)=\rho(t) \frac{r(t)\left(z^{\prime}(t)\right)^{\alpha}}{z^{\alpha}(\sigma(t))} .
$$

Clearly $\omega(t)>0$ and

$$
\omega^{\prime}(t)=\rho^{\prime}(t) \frac{r(t)\left(z^{\prime}(t)\right)^{\alpha}}{z^{\alpha}(\sigma(t))}+\rho(t) \frac{\left(r(t)\left(z^{\prime}(t)\right)^{\alpha}\right)^{\prime}}{z^{\alpha}(\sigma(t))}-\alpha \rho(t) \frac{r(t)\left(z^{\prime}(t)\right)^{\alpha} z^{\prime}(\sigma(t)) \sigma^{\prime}(t)}{z^{\alpha+1}(\sigma(t))} .
$$

From $\sigma(t) \leq t$ and from the monotonicity of $r(t) \Phi\left(z^{\prime}(t)\right)$ we have

$$
z^{\prime}(\sigma(t)) \geq\left(\frac{r(t)}{r(\sigma(t))}\right)^{1 / \alpha} z^{\prime}(t)
$$

and combining these computations we get

$$
\omega^{\prime}(t) \leq \frac{\rho^{\prime}(t)}{\rho(t)} \omega(t)+\rho(t) \frac{\left(r(t)\left(z^{\prime}(t)\right)^{\alpha}\right)^{\prime}}{z^{\alpha}(\sigma(t))}-\frac{\alpha \sigma^{\prime}(t)}{\rho^{1 / \alpha}(t) r^{1 / \alpha}(\sigma(t))} \omega^{\frac{\alpha+1}{\alpha}}(t) .
$$

Further we define

$$
v(t)=\rho(t) \frac{r(\tau(t))\left(z^{\prime}(\tau(t))\right)^{\alpha}}{z^{\alpha}(\sigma(t))},
$$

we use the obvious fact $v(t)>0$ and differentiate

$$
\begin{aligned}
v^{\prime}(t)= & \rho^{\prime}(t) \frac{r(\tau(t))\left(z^{\prime}(\tau(t))\right)^{\alpha}}{z^{\alpha}(\sigma(t))}+\rho(t) \frac{\left(r(\tau(t))\left(z^{\prime}(\tau(t))\right)^{\alpha}\right)^{\prime}}{z^{\alpha}(\sigma(t))} \\
& -\alpha \rho(t) \frac{\left.r(\tau(t))\left(z^{\prime}(\tau(t))\right)\right)^{\alpha} z^{\prime}(\sigma(t)) \sigma^{\prime}(t)}{z^{\alpha+1}(\sigma(t))} .
\end{aligned}
$$

Using the monotonicity of $r(t) \Phi\left(z^{\prime}(t)\right)$ and $\sigma(t) \leq \tau(t)$ we have

$$
z^{\prime}(\sigma(t)) \geq\left(\frac{r(\tau(t))}{r(\sigma(t))}\right)^{1 / \alpha} z^{\prime}(\tau(t))
$$

and hence

$$
v^{\prime}(t) \leq \frac{\rho^{\prime}(t)}{\rho(t)} v(t)+\rho(t) \frac{\left(r(\tau(t))\left(z^{\prime}(\tau(t))\right)^{\alpha}\right)^{\prime}}{z^{\alpha}(\sigma(t))}-\frac{\alpha \sigma^{\prime}(t)}{\rho^{1 / \alpha}(t) r^{1 / \alpha}(\sigma(t))} v^{\frac{\alpha+1}{\alpha}}(t) .
$$


Multiplying (17) by $l^{\alpha-1}$, (19) by $\left(l^{*}\right)^{\alpha-1} \frac{p^{\alpha}(\sigma(t)) \varphi(t)}{\tau^{\prime}(t)}$, adding the resulting inequalities and using (10), we get

$$
\begin{aligned}
& l^{\alpha-1} \omega^{\prime}(t)+\left(l^{*}\right)^{\alpha-1} \frac{p^{\alpha}(\sigma(t)) \varphi(t)}{\tau^{\prime}(t)} v^{\prime}(t) \\
& \leq-\rho(t) Q(t)+l^{\alpha-1}\left[\frac{\rho^{\prime}(t)}{\rho(t)} \omega(t)-\frac{\alpha \sigma^{\prime}(t)}{\rho^{1 / \alpha}(t) r^{1 / \alpha}(\sigma(t))} \omega^{\frac{\alpha+1}{\alpha}}(t)\right] \\
& \quad+\left(l^{*}\right)^{\alpha-1}\left[\frac{p^{\alpha}(\sigma(t)) \varphi(t)}{\tau^{\prime}(t)} \frac{\rho^{\prime}(t)}{\rho(t)} v(t)-\frac{p^{\alpha}(\sigma(t)) \varphi(t)}{\tau^{\prime}(t)} \frac{\alpha \sigma^{\prime}(t)}{\rho^{1 / \alpha}(t) r^{1 / \alpha}(\sigma(t))} v^{\frac{\alpha+1}{\alpha}}(t)\right] .
\end{aligned}
$$

Using the product rule for derivatives we obtain

$$
\begin{aligned}
l^{\alpha-1} \omega^{\prime}(t)+\left(l^{*}\right)^{\alpha-1}\left(\frac{p^{\alpha}(\sigma(t)) \varphi(t)}{\tau^{\prime}(t)} v(t)\right)^{\prime} \\
\leq-\rho(t) Q(t)+l^{\alpha-1}\left[\frac{\rho^{\prime}(t)}{\rho(t)} \omega(t)-\frac{\alpha \sigma^{\prime}(t)}{\rho^{1 / \alpha}(t) r^{1 / \alpha}(\sigma(t))} \omega^{\frac{\alpha+1}{\alpha}}(t)\right] \\
+\left(l^{*}\right)^{\alpha-1}\left[\left(\frac{p^{\alpha}(\sigma(t)) \varphi(t)}{\tau^{\prime}(t)} \frac{\rho^{\prime}(t)}{\rho(t)}+\left(\frac{p^{\alpha}(\sigma(t)) \varphi(t)}{\tau^{\prime}(t)}\right)\right) v(t)\right. \\
\left.-\frac{p^{\alpha}(\sigma(t)) \varphi(t)}{\tau^{\prime}(t)} \frac{\alpha \sigma^{\prime}(t)}{\rho^{1 / \alpha}(t) r^{1 / \alpha}(\sigma(t))} v^{\frac{\alpha+1}{\alpha}}(t)\right]
\end{aligned}
$$

and Lemma 1 implies

$$
\begin{aligned}
l^{\alpha-1} \omega^{\prime}(t)+\left(l^{*}\right)^{\alpha-1}\left(\frac{p^{\alpha}(\sigma(t)) \varphi(t)}{\tau^{\prime}(t)} v(t)\right)^{\prime} \\
\leq-\rho(t) Q(t)+l^{\alpha-1} \frac{\alpha^{\alpha}}{(\alpha+1)^{\alpha+1}}\left(\frac{\rho_{+}^{\prime}(t)}{\rho(t)}\right)^{\alpha+1} \frac{\rho(t) r(\sigma(t))}{\alpha^{\alpha}\left(\sigma^{\prime}(t)\right)^{\alpha}} \\
\quad+\left(l^{*}\right)^{\alpha-1} \frac{\alpha^{\alpha}}{(\alpha+1)^{\alpha+1}}\left(\frac{p^{\alpha}(\sigma(t)) \varphi(t)}{\tau^{\prime}(t)} \frac{\rho^{\prime}(t)}{\rho(t)}+\left(\frac{p^{\alpha}(\sigma(t)) \varphi(t)}{\tau^{\prime}(t)}\right)^{\prime}\right)_{+}^{\alpha+1} \\
\quad \times \frac{\left(\tau^{\prime}(t)\right)^{\alpha}}{p^{\alpha^{2}}(\sigma(t)) \varphi^{\alpha}(t)} \frac{\rho(t) r(\sigma(t))}{\alpha^{\alpha}\left(\sigma^{\prime}(t)\right)^{\alpha}} .
\end{aligned}
$$

Integrating from $t_{2}$ to $t$

$$
\begin{aligned}
l^{\alpha-1} \omega(t) & -l^{\alpha-1} \omega\left(t_{2}\right)+\left(l^{*}\right)^{\alpha-1} \frac{p^{\alpha}(\sigma(t)) \varphi(t)}{\tau^{\prime}(t)} v(t)-\left(l^{*}\right)^{\alpha-1} \frac{p^{\alpha}\left(\sigma\left(t_{2}\right)\right) \varphi\left(t_{2}\right)}{\tau^{\prime}\left(t_{2}\right)} v\left(t_{2}\right) \\
\leq & -\int_{t_{2}}^{t} \rho(s) Q(s)-\frac{1}{(\alpha+1)^{\alpha+1}} \frac{\rho(s) r(\sigma(s))}{\left(\sigma^{\prime}(s)\right)^{\alpha}} \\
& \times\left[l^{\alpha-1}\left(\frac{\rho_{+}^{\prime}(s)}{\rho(s)}\right)^{\alpha+1}+\left(l^{*}\right)^{\alpha-1} \frac{p^{\alpha}(\sigma(s)) \varphi(s)}{\tau^{\prime}(s)}\right. \\
& \left.\times\left(\frac{\rho^{\prime}(s)}{\rho(s)}+\left(\frac{p^{\alpha}(\sigma(s)) \varphi(s)}{\tau^{\prime}(s)}\right)^{\prime} \frac{\tau^{\prime}(s)}{p^{\alpha}(\sigma(s)) \varphi(s)}\right)_{+}^{\alpha+1}\right] \mathrm{d} s .
\end{aligned}
$$

Multiplying by -1 and taking into account the fact that both $\omega(t)$ and $\frac{p^{\alpha}(\sigma(t)) \varphi(t)}{\tau^{\prime}(t)} v(t)$ are nonnegative we get a finite upper bound for the integral from (15), which contradicts (15). 
Remark 1 Under the conditions $l=l^{*}=2, \varphi(t)=1$ we can obtain [3, Theorem 3.1] as a corollary of Theorem 1 , since the inequality

$$
\left(\frac{\rho^{\prime}(s)}{\rho(s)}+\left(\frac{p^{\alpha}(\sigma(s)) \varphi(s)}{\tau^{\prime}(s)}\right)^{\prime} \frac{\tau^{\prime}(s)}{p^{\alpha}(\sigma(s)) \varphi(s)}\right)_{+} \leq \frac{\rho_{+}^{\prime}(s)}{\rho(s)}+\left(\left(\frac{p^{\alpha}(\sigma(s)) \varphi(s)}{\tau^{\prime}(s)}\right)^{\prime} \frac{\tau^{\prime}(s)}{p^{\alpha}(\sigma(s)) \varphi(s)}\right)_{+}
$$

holds.

The following corollary is in fact a variant of Theorem 1 if $p(t)$ is bounded above by a nonnegative number and $\tau^{\prime}(t)$ is bounded below by a positive number. Since (15) is not simply monotone with respect to $p(t)$ and $\tau(t)$, we have to include the corresponding estimates in the opening part of the proof.

Corollary 1 Suppose that (7), (14), $\sigma(t) \leq t$ and $\sigma(t) \leq \tau(t)$ are satisfied and there exist constants $p_{0} \geq 0$ and $\tau_{0}>0$ such that $p(t) \leq p_{0}<\infty$ and $\tau^{\prime}(t) \geq \tau_{0}$. If there exist positive mutually conjugate numbers $l, l^{*}$, and positive functions $\rho(t), \varphi(t)$ such that

$$
\begin{aligned}
& \limsup _{t \rightarrow \infty} \int_{t_{0}}^{t} \rho(s) Q(s)-\frac{1}{(\alpha+1)^{\alpha+1}} \frac{\rho(s) r(\sigma(s))}{\left(\sigma^{\prime}(s)\right)^{\alpha}} \\
& \quad \times\left[l^{\alpha-1}\left(\frac{\rho_{+}^{\prime}(s)}{\rho(s)}\right)^{\alpha+1}+\left(l^{*}\right)^{\alpha-1} \frac{p_{0}^{\alpha} \varphi(s)}{\tau_{0}}\left(\frac{\rho^{\prime}(s)}{\rho(s)}+\left(\frac{p_{0}^{\alpha} \varphi(s)}{\tau_{0}}\right)^{\prime} \frac{\tau_{0}}{p_{0}^{\alpha} \varphi(s)}\right)_{+}^{\alpha+1}\right] \mathrm{d} s=\infty,
\end{aligned}
$$

then (1) is oscillatory.

Proof The proof is the same as the proof of Theorem 1, we just use (12) instead of (10) and in the remaining part of the proof we replace $p(t)$ by $p_{0}$ and $\tau^{\prime}(t)$ by $\tau_{0}$.

Example 1 For the Euler type equation (2) with $0<\lambda_{2} \leq \lambda_{1}<1$ we have $q(t)=\frac{\beta}{t^{\alpha+1}}, r(t)=$ $1, \sigma(t)=\lambda_{2} t, \sigma^{\prime}(t)=\lambda_{2}, \tau(t)=\lambda_{1} t, \tau^{\prime}(t)=\tau_{0}=\lambda_{1}, q(\tau(t))=\frac{\beta}{\lambda_{1}^{\alpha+1} t^{\alpha+1}}$. Denote $\varphi(t)=\lambda_{1}^{\alpha+1}$ and $\rho(t)=t^{\alpha}$. With this setting we have $q(t)=\varphi(t) q(\tau(t))$ and hence $Q(t)=q(t)$. Further $\rho(t) Q(t)=\frac{\beta}{t}, \frac{\rho^{\prime}(t)}{\rho(t)}=\frac{\alpha}{t}, \frac{p_{0}^{\alpha} \varphi(t)}{\tau_{0}}=\left(p_{0} \lambda_{1}\right)^{\alpha}$ and $(20)$ becomes

$$
\left[\beta-\left(\frac{\alpha}{\alpha+1}\right)^{\alpha+1} \frac{1}{\lambda_{2}^{\alpha}}\left[l^{\alpha-1}+\left(l^{*}\right)^{\alpha-1} p_{0}^{\alpha} \lambda_{1}^{\alpha}\right]\right] \limsup _{t \rightarrow \infty} \int^{t} \frac{1}{s} \mathrm{~d} s=\infty
$$

and (2) is oscillatory if

$$
\beta>\left(\frac{\alpha}{\alpha+1}\right)^{\alpha+1} \frac{1}{\lambda_{2}^{\alpha}}\left[l^{\alpha-1}+\left(l^{*}\right)^{\alpha-1} p_{0}^{\alpha} \lambda_{1}^{\alpha}\right]
$$

Note that if $l=l^{*}=2$, then this condition becomes

$$
\beta>\left(\frac{\alpha}{\alpha+1}\right)^{\alpha+1} \frac{2^{\alpha-1}}{\lambda_{2}^{\alpha}}\left[1+p_{0}^{\alpha} \lambda_{1}^{\alpha}\right]
$$

and since for $\lambda_{1}<1$ we have $\lambda_{1}^{\alpha}<1<\frac{1}{\lambda_{1}}$, this oscillation constant is smaller than the oscillation constant from (3). 
Further, if $p_{0}=0$ and $\lambda_{1}=1=\lambda_{2}$, then (1) becomes (5). Condition (21) becomes

$$
\beta>\left(\frac{\alpha}{\alpha+1}\right)^{\alpha+1} l^{\alpha-1}
$$

and, since $l>1$ is arbitrary, we get

$$
\beta>\left(\frac{\alpha}{\alpha+1}\right)^{\alpha+1}
$$

which is well known to be an optimal and non-improvable oscillation constant for (5). In this sense we consider our result as reasonably sharp.

Finally, taking into account that $l^{*}=\frac{l}{l-1}$, condition (21) becomes

$$
\beta>\left(\frac{\alpha}{\alpha+1}\right)^{\alpha+1} \frac{1}{\lambda_{2}^{\alpha}}\left[l^{\alpha-1}+\left(\frac{l}{l-1}\right)^{\alpha-1} p_{0}^{\alpha} \lambda_{1}^{\alpha}\right]
$$

A simple computation shows that the function

$$
f(l)=l^{\alpha-1}+\left(\frac{l}{l-1}\right)^{\alpha-1} p_{0}^{\alpha} \lambda_{1}^{\alpha}, \quad l>1
$$

satisfies

$$
f^{\prime}(l)=(\alpha-1) l^{\alpha-2}\left[1-\frac{1}{(l-1)^{\alpha}} p_{0}^{\alpha} \lambda_{1}^{\alpha}\right]
$$

and has a global minimum at $l=1+p_{0} \lambda_{1}$. Thus the choice $l=1+p_{0} \lambda_{1}$ in (21) produces the smallest oscillation constant

$$
\beta>\left(\frac{\alpha}{\alpha+1}\right)^{\alpha+1} \frac{\left(1+p_{0} \lambda_{1}\right)^{\alpha}}{\lambda_{2}^{\alpha}}
$$

Example 2 Baculíková et al. [5, Example 2.1] considered the equation

$$
\left(t\left|z^{\prime}(t)\right| z^{\prime}(t)\right)^{\prime}+\frac{b}{t^{2}}|x(\beta t)| x(\beta t)=0
$$

with $z(t)=x(t)+p_{0} x(\omega t), p_{0}>0, b>0$ and $0<\beta<1$. They proved that under the condition $\beta<\omega<1$ (23) is oscillatory if

$$
b \ln \frac{\omega}{\beta}>\frac{\omega+p_{0}^{2}}{2 \beta e \omega}
$$

(note that this condition is misprinted in [5]). This condition naturally produces poor oscillation constant if $\beta$ is close to $\omega$. In our notation we have $\alpha=2, q(t)=b / t^{2}, r(t)=t$, $\sigma(t)=\beta t, \sigma^{\prime}(t)=\beta, \tau(t)=\omega t, \tau^{\prime}(t)=\tau_{0}=\omega$. We choose $\varphi(t)=\omega^{2}$ and $\rho(t)=t$. Thus (20) takes the form

$$
\left[b-\frac{l+l^{*} p_{0}^{2} \omega}{3^{3} \beta}\right] \lim _{t \rightarrow \infty} \int_{t_{0}}^{t} \frac{1}{s} \mathrm{~d} s=\infty .
$$


Figure 1 Bounds for oscillation constant $b$ from (23) for $p_{0}=0.5, \omega=0.75$.

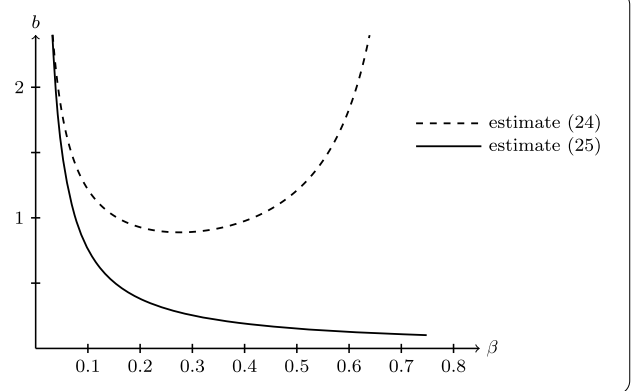

Taking into account that $l^{*}=\frac{l}{l-1}$ and that the function $l+l^{*} p_{0}^{2} \omega$ has a local minimum $\left(1+p_{0} \sqrt{\omega}\right)^{2}$ at the point $l=1+p_{0} \sqrt{\omega}$, we find that (23) is oscillatory if

$$
b>\frac{\left(1+p_{0} \sqrt{\omega}\right)^{2}}{27 \beta} .
$$

This condition completes condition (24). It is possible to find constants $\omega$ and $\beta$ for which (25) is better than (24), as well as constants where the opposite is true. The fact that both estimates depend heavily on the parameters is illustrated by Figure 1.

The following corollary suggests another modification of the proof of Theorem 1: we replace condition (7) by weaker condition (8) and add conditions which ensure that $x$ possesses the same type of monotonicity as $z$.

Corollary 2 Suppose that $p(t) \equiv p_{0},(8),(14), \sigma(t) \leq t$ and $\sigma(t) \leq \tau(t)$ hold.If $(20)$ holds for some mutually conjugate numbers $l, l^{*}$ and positive functions $\rho(t), \varphi(t)$, then every solution of (1) is either oscillatory, or the first derivative of this solution is oscillatory.

Proof Suppose, by contradiction, that the assumptions are satisfied and $x$ is an eventually positive solution of (1) such that $x^{\prime}(t)$ is not oscillatory.

We proceed as in Theorem 1 with modifications mentioned in the proof of Corollary 1. To ensure that Lemma 3 can be applied even though (7) need not to hold note that from the fact that $z$ is eventually increasing, $p(t)$ constant and $x(t)$ not oscillatory we conclude easily that $x$ is also eventually increasing.

In the following example we show an application of Corollary 2 to the equation where $\sigma(\tau(t)) \neq \tau(\sigma(t))$.

Example 3 Consider the equation

$$
\left[\Phi\left(\left(x(t)+p_{0} x\left(t-\lambda_{1}\right)\right)^{\prime}\right)\right]^{\prime}+\frac{\beta}{t^{\alpha+1}} \Phi\left(x\left(\lambda_{2} t\right)\right)=0
$$

with $0 \leq p_{0}, \alpha \geq 1, \beta>0, \lambda_{1}>0, \lambda_{2} \in(0,1)$. We have $q(t)=\beta / t^{\alpha+1}, \sigma(t)=\lambda_{2} t, \sigma^{\prime}(t)=\lambda_{2}$, $\tau(t)=t-\lambda_{1}, \tau^{\prime}(t)=\tau_{0}=1, \sigma(\tau(t))=\lambda_{2} t-\lambda_{2} \lambda_{1} \geq \lambda_{2} t-\lambda_{1}=\tau(\sigma(t)), \sigma(t) \leq \tau(t)$ for large $t$ and $q(\tau(t)) \geq q(t)$. We choose $\varphi(t)=1$ and $\rho(t)=t^{\alpha}$. With this setting the condition (20) takes the form

$$
\left[\beta-\left(\frac{\alpha}{\alpha+1}\right)^{\alpha+1} \frac{1}{\lambda_{2}^{\alpha}}\left(l^{\alpha-1}+\left(l^{*}\right)^{\alpha-1} p_{0}^{\alpha}\right)\right] \lim _{t \rightarrow \infty} \ln \frac{t}{t_{0}}=\infty .
$$


Using this computation and using the fact that the function $l^{\alpha-1}+\left(l^{*}\right)^{\alpha-1} p_{0}^{\alpha}$ takes global minimum on $l \in(1, \infty)$ for $l=1+p_{0}$ and $l^{*}=1+1 / p_{0}$ we see that the condition

$$
\beta>\left(\frac{\alpha}{\alpha+1}\right)^{\alpha+1} \frac{\left(1+p_{0}\right)^{\alpha}}{\lambda_{2}^{\alpha}}
$$

guarantees that either every solution or derivative of every solution of the equation is oscillatory.

In the following theorem we drop the condition $\sigma(t) \leq \tau(t)$ and use the opposite $\sigma(t) \geq$ $\tau(t)$. In this case we modify the denominator in the Riccati type substitutions (16) and (18).

Theorem 2 Suppose that (7), (14), $\tau(t) \leq t$ and $\sigma(t) \geq \tau(t)$ hold. Further suppose that there exist positive mutually conjugate numbers $l, l^{*}$ and positive functions $\rho \in C^{1}\left(\left[t_{0}, \infty\right), \mathbb{R}^{+}\right)$, $\varphi(t)$ such that

$$
\begin{aligned}
& \limsup _{t \rightarrow \infty} \int_{t_{0}}^{t} \rho(s) Q(s)-\frac{1}{(\alpha+1)^{\alpha+1}} \frac{\rho(s) r(\tau(s))}{\left(\tau^{\prime}(s)\right)^{\alpha}} \\
& \quad \times\left[l^{\alpha-1}\left(\frac{\rho_{+}^{\prime}(s)}{\rho(s)}\right)^{\alpha+1}+\left(l^{*}\right)^{\alpha-1} \frac{p^{\alpha}(\sigma(s)) \varphi(s)}{\tau^{\prime}(s)}\right. \\
& \left.\quad \times\left(\frac{\rho^{\prime}(s)}{\rho(s)}+\left(\frac{p^{\alpha}(\sigma(s)) \varphi(s)}{\tau^{\prime}(s)}\right)^{\prime} \frac{\tau^{\prime}(s)}{p^{\alpha}(\sigma(s)) \varphi(s)}\right)_{+}^{\alpha+1}\right] \mathrm{d} s=\infty .
\end{aligned}
$$

Then (1) is oscillatory.

Proof Suppose, by contradiction, that all the conditions are satisfied and an eventually positive solution $x(t)$ of (1) exists. As in the proof of Theorem 1, we can show that $r(t) \Phi\left(z^{\prime}(t)\right)$ is decreasing eventually and $z^{\prime}(t)$ increasing eventually. Let us work on the interval $\left(t_{2}, \infty\right)$ where $t_{2}$ is such that

$$
\min \left\{x(t), x(\tau(t)), x(\sigma(t)), x(\tau(\sigma(t))), z^{\prime}(t), z^{\prime}(\tau(t)), z^{\prime}(\sigma(t)), z^{\prime}(\tau(\sigma(t)))\right\}>0
$$

for every $t>t_{2}$.

Define

$$
\omega(t)=\rho(t) \frac{r(t)\left(z^{\prime}(t)\right)^{\alpha}}{z^{\alpha}(\tau(t))}
$$

As in the proof of Theorem 1, we have $\omega(t)>0$ and

$$
\omega^{\prime}(t)=\rho^{\prime}(t) \frac{r(t)\left(z^{\prime}(t)\right)^{\alpha}}{z^{\alpha}(\tau(t))}+\rho(t) \frac{\left(r(t)\left(z^{\prime}(t)\right)^{\alpha}\right)^{\prime}}{z^{\alpha}(\tau(t))}-\alpha \rho(t) \frac{r(t)\left(z^{\prime}(t)\right)^{\alpha} z^{\prime}(\tau(t)) \tau^{\prime}(t)}{z^{\alpha+1}\left(\tau^{\prime}(t)\right)} .
$$

From $\tau(t) \leq t$ and from the monotonicity of $r(t) \Phi\left(z^{\prime}(t)\right)$ we have

$$
z^{\prime}(\tau(t)) \geq\left(\frac{r(t)}{r(\tau(t))}\right)^{1 / \alpha} z^{\prime}(t)
$$


and combining these computations we get

$$
\omega^{\prime}(t) \leq \frac{\rho^{\prime}(t)}{\rho(t)} \omega(t)+\rho(t) \frac{\left(r(t)\left(z^{\prime}(t)\right)^{\alpha}\right)^{\prime}}{z^{\alpha}(\tau(t))}-\frac{\alpha \tau^{\prime}(t)}{\rho^{1 / \alpha}(t) r^{1 / \alpha}(\tau(t))} \omega^{\frac{\alpha+1}{\alpha}}(t) .
$$

Further we define

$$
v(t)=\rho(t) \frac{r(\tau(t))\left(z^{\prime}(\tau(t))\right)^{\alpha}}{z^{\alpha}(\tau(t))},
$$

differentiate

$$
\begin{aligned}
v^{\prime}(t)= & \rho^{\prime}(t) \frac{r(\tau(t))\left(z^{\prime}(\tau(t))\right)^{\alpha}}{z^{\alpha}(\tau(t))}+\rho(t) \frac{\left(r(\tau(t))\left(z^{\prime}(\tau(t))\right)^{\alpha}\right)^{\prime}}{z^{\alpha}(\tau(t))} \\
& -\alpha \rho(t) \frac{r(\tau(t))\left(z^{\prime}(\tau(t))\right)^{\alpha} z^{\prime}(\tau(t)) \tau^{\prime}(t)}{z^{\alpha+1}(\tau(t))}
\end{aligned}
$$

and conclude

$$
v^{\prime}(t)=\frac{\rho^{\prime}(t)}{\rho(t)} v(t)+\rho(t) \frac{\left(r(\tau(t))\left(z^{\prime}(\tau(t))\right)^{\alpha}\right)^{\prime}}{z^{\alpha}(\tau(t))}-\frac{\alpha \tau^{\prime}(t)}{\rho^{1 / \alpha}(t) r^{1 / \alpha}(\tau(t))} v^{\frac{\alpha+1}{\alpha}}(t) .
$$

Similarly as in the proof of Theorem 1 and using the fact that monotonicity of $z(t)$ and inequality $\sigma(t) \geq \tau(t)$ imply $Q(t) \frac{z(\sigma(t))}{z(\tau(t))} \geq Q(t)$, we get

$$
\begin{aligned}
& l^{\alpha-1} \omega^{\prime}(t)+\left(l^{*}\right)^{\alpha-1} \frac{p^{\alpha}(\sigma(t)) \varphi(t)}{\tau^{\prime}(t)} v^{\prime}(t) \\
& \leq--\rho(t) Q(t)+l^{\alpha-1}\left[\frac{\rho^{\prime}(t)}{\rho(t)} \omega(t)-\frac{\alpha \tau^{\prime}(t)}{\rho^{1 / \alpha}(t) r^{1 / \alpha}(\tau(t))} \omega^{\frac{\alpha+1}{\alpha}}(t)\right] \\
& \quad+\left(l^{*}\right)^{\alpha-1}\left[\frac{p^{\alpha}(\sigma(t)) \varphi(t)}{\tau^{\prime}(t)} \frac{\rho^{\prime}(t)}{\rho(t)} v(t)-\frac{p^{\alpha}(\sigma(t)) \varphi(t)}{\tau^{\prime}(t)} \frac{\alpha \tau^{\prime}(t)}{\rho^{1 / \alpha}(t) r^{1 / \alpha}(\tau(t))} v^{\frac{\alpha+1}{\alpha}}(t)\right] .
\end{aligned}
$$

The remaining part of the proof is the same as in Theorem 1.

Remark 2 Similarly as in Remark 1, [3, Theorem 3.3] is a corollary of Theorem 2.

Corollary 3 Suppose that (7), (14), $\tau(t) \leq t$ and $\sigma(t) \geq \tau(t)$ hold. Furthermore, suppose that there exist constants $p_{0} \geq 0$ and $\tau_{0}>0$ such that $p(t) \leq p_{0}<\infty$ and $\tau^{\prime}(t) \geq \tau_{0}$. If there exist positive mutually conjugate numbers $l, l^{*}$, and positive functions $\rho(t), \varphi(t)$ such that

$$
\begin{aligned}
& \limsup _{t \rightarrow \infty} \int_{t_{0}}^{t} \rho(s) Q(s)-\frac{1}{(\alpha+1)^{\alpha+1}} \frac{\rho(s) r(\tau(s))}{\left(\tau^{\prime}(s)\right)^{\alpha}} \\
& \quad \times\left[l^{\alpha-1}\left(\frac{\rho_{+}^{\prime}(s)}{\rho(s)}\right)^{\alpha+1}+\left(l^{*}\right)^{\alpha-1} \frac{p_{0}^{\alpha} \varphi(s)}{\tau_{0}}\left(\frac{\rho^{\prime}(s)}{\rho(s)}+\left(\frac{p_{0}^{\alpha} \varphi(s)}{\tau_{0}}\right)^{\prime} \frac{\tau_{0}}{p_{0}^{\alpha} \varphi(s)}\right)_{+}^{\alpha+1}\right] \mathrm{d} s=\infty,
\end{aligned}
$$

then (1) is oscillatory.

Proof The proof is he same as the proof of Corollary 1. We just use Theorem 2 instead of Theorem 1. 
Example 4 Consider (2) with $\lambda_{2} \geq \lambda_{1}$. We choose the functions $\rho$ and $\varphi$ as in Example 1 and find that (1) is oscillatory if

$$
\beta>\left(\frac{\alpha}{\alpha+1}\right)^{\alpha+1} \frac{\left(1+p_{0} \lambda_{1}\right)^{\alpha}}{\lambda_{1}^{\alpha}}
$$

Let us compare this result with (4). The inequalities $\alpha \geq 1, p_{0} \geq 0$, and $\lambda_{1}<1$ imply

$$
2^{\alpha-1}+2^{\alpha-1} \frac{p_{0}^{\alpha}}{\lambda_{1}} \geq 2^{\alpha-1}+2^{\alpha-1} p_{0}^{\alpha} \lambda_{1}^{\alpha}=f(2) \geq\left(1+p_{0} \lambda_{1}\right)^{\alpha},
$$

where $f$ is defined by $(22)$ and $\left(1+p_{0} \lambda_{1}\right)^{\alpha}$ is a global minimum of $f$ on $(1, \infty)$. Hence

$$
\frac{2^{\alpha-1} \alpha^{\alpha+1}}{(\alpha+1)^{\alpha+1} \lambda_{1}^{\alpha}}\left(1+\frac{p_{0}}{\lambda_{1}}\right) \geq\left(\frac{\alpha}{\alpha+1}\right)^{\alpha+1} \frac{\left(1+p_{0} \lambda_{1}\right)^{\alpha}}{\lambda_{1}^{\alpha}}
$$

and (27) is sharper than (4).

The following corollary is a variant of Corollary 2 for $\sigma(t) \geq \tau(t)$.

Corollary 4 Suppose that $p(t) \equiv p_{0},(8),(14), \tau(t) \leq t$ and $\sigma(t) \geq \tau(t)$ hold. If (26) holds for some mutually conjugate numbers $l, l^{*}$ and positive functions $\rho(t), \varphi(t)$, then every solution of (1) is either oscillatory, or the first derivative of this solution is oscillatory.

Proof The proof is the same as the proof of Corollary 2; we only replace Theorem 1 by Theorem 2 and Corollary 1 by Corollary 3 .

Remark 3 There are two main approaches how to handle Riccati type transformation in the oscillation theory of neutral differential equations. The first applies if $0 \leq p(t)<1$ and the shift in the differential term is handled by utilizing the estimate $z(t)(1-p(t)) \leq$ $x(t)$; see $e . g$. [7-9]. Thus the results of this type depend on term $(1-p(\sigma(t)))$. Another frequent approach which has been used in $[3,10]$ and also in this paper is summing up the equation at $t$ and $\tau(t)$ and working with the resulting sum. Since it is necessary to take out common factor, the oscillation criteria usually contain term $\min \{q(t), q(\tau(t))\}$. Since both $q(t)$ and $q(\tau(t))$ may differ significantly, we developed in this paper a method which replaces this term with the term $\min \{q(t), \varphi(t) q(\tau(t))\}$, where the function $\varphi(t)$ is in some sense arbitrary and may have influence on the final oscillation criterion. We also showed on examples in previous section that this idea produces nonempty extension of known results. We conjecture that a similar idea can be used to obtain new results also in the case of a series of papers by Baculíková and Džurina [5, 11, 12], where a sum of two equations (in the original variable and in the shifted variable) is used to derive a certain first-order delay differential equation and the oscillation criteria are formulated in terms of this first-order equation. However, this idea exceeds the scope of this paper and will be examined in other research.

\section{Conclusion}

New oscillation theorems for second-order half-linear differential equations have been obtained. The novelty is in the point that we employed general linear combination based 
on conjugate numbers $l$ and $l^{*}$ rather than its special case $l=2=l^{*}$ considered in the other papers devoted to this problem and also included a parameter $\varphi(t)$ which plays a role when taking minimum of $q(t)$ and $q(\tau(t))$. These extensions are capable to produce sharper results than the results published in the literature as has been shown on examples. As a byproduct we also relaxed in Corollaries 2 and 4 the usual requirement that the composition of delays is commutative. This makes our results applicable to equations with combined constant and proportional delays.

Competing interests

The authors declare that they have no competing interests.

Authors' contributions

The paper has been prepared by both authors. Both authors have made the same contribution and approved the final manuscript.

\section{Acknowledgements}

This research was supported by the Grant P201/10/1032 of the Czech Science Foundation.

Received: 10 January 2014 Accepted: 1 April 2014 Published: 10 Apr 2014

References

1. Burton, TA, Purnaras, IK: A unification theory of Krasnoselskii for differential equations. Nonlinear Anal. 89, 121-133 (2013)

2. Smith, FE: Population dynamics in Daphnia magna and a new model for population growth. Ecology 44(4), 651-663 (1963)

3. Sun, $\mathrm{S}, \mathrm{Li}, \mathrm{T}, \mathrm{Han}, \mathrm{Z}, \mathrm{Li}, \mathrm{H}$ : Oscillation theorems for second order quasilinear neutral functional differential equations. Abstr. Appl. Anal. 2012, Article ID 819342 (2012)

4. Došlý, O, Rehák, P: Half-Linear Differential Equations. North-Holland Mathematics Studies, vol. 202. Elsevier, Amsterdam (2005)

5. Baculíková, B, Li, T, Džurina, J: Oscillation theorems for second-order superlinear neutral differential equations. Math Slovaca 63, 123-134 (2013)

6. Li, T, Han, Z, Zhang, C, Li, H: Oscillation criteria for second-order superlinear neutral differential equations. Abstr. Appl. Anal. 2011, Article ID 367541 (2011)

7. Dong, JG: Oscillation behavior of second order nonlinear neutral differential equations with deviating arguments Comput. Math. Appl. 59, 3710-3717 (2010)

8. Fišnarová, S, Mařík, R: Oscillation of half-linear differential equations with delay. Abstr. Appl. Anal. 2013, Article ID 583147 (2013)

9. Hasanbulli, M, Rogovchenko, Y: Oscillation criteria for second order nonlinear neutral differential equations. Appl. Math. Comput. 215, 4392-4399 (2010)

10. Han, Z, Li, T, Sun, S, Chen, W: Oscillation criteria for second-order nonlinear neutral delay differential equations. Adv. Differ. Equ. 2010, Article ID 763278 (2010)

11. Baculíková, B, Džurina, J: Oscillation theorems for second-order neutral differential equations. Comput. Math. Appl. 61, 94-99 (2011)

12. Baculíková, B, Džurina, J: Oscillation theorems for second-order nonlinear neutral differential equations. Comput. Math. Appl. 62, 4472-4478 (2011)

10.1186/1687-2770-2014-83

Cite this article as: Fišnarová and Mařík: Oscillation criteria for neutral second-order half-linear differential equations with applications to Euler type equations. Boundary Value Problems 2014, 2014:83

\section{Submit your manuscript to a SpringerOpen ${ }^{0}$ journal and benefit from:}

- Convenient online submission

- Rigorous peer review

Immediate publication on acceptance

- Open access: articles freely available online

- High visibility within the field

- Retaining the copyright to your article 